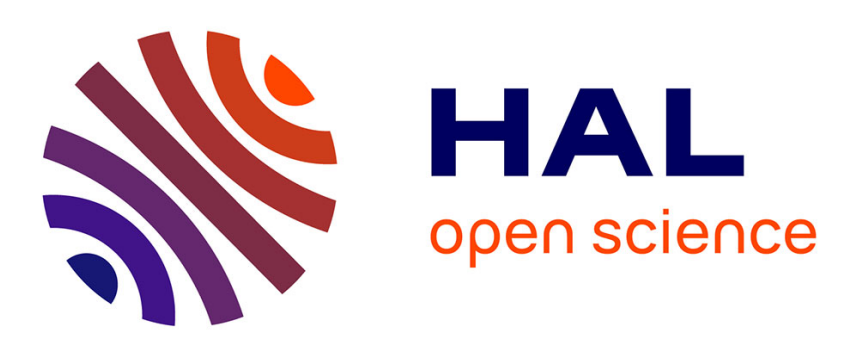

\title{
On the Use of Automatic Cuts Algorithm for T0 - T $-\Phi$ Formulation in Nondestructive Testing by Eddy Current
}

\author{
Anh-Tuan Phung, Patrice Labie, Olivier Chadebec, Yann Le Floch, Gerard
}

Meunier

\section{- To cite this version:}

Anh-Tuan Phung, Patrice Labie, Olivier Chadebec, Yann Le Floch, Gerard Meunier. On the Use of Automatic Cuts Algorithm for T0 $-\mathrm{T}-\Phi$ Formulation in Nondestructive Testing by Eddy Current. Studies in Computational Intelligence, 2008, 119, pp 55-62. 10.1007/978-3-540-78490-6_7 . hal00355148

\author{
HAL Id: hal-00355148 \\ https://hal.science/hal-00355148
}

Submitted on 11 Feb 2021

HAL is a multi-disciplinary open access archive for the deposit and dissemination of scientific research documents, whether they are published or not. The documents may come from teaching and research institutions in France or abroad, or from public or private research centers.
L'archive ouverte pluridisciplinaire HAL, est destinée au dépôt et à la diffusion de documents scientifiques de niveau recherche, publiés ou non, émanant des établissements d'enseignement et de recherche français ou étrangers, des laboratoires publics ou privés. 


\title{
On the Use of Automatic Cuts Algorithm for $\mathbf{T}_{\mathbf{0}}-\mathbf{T}-\Phi$ Formulation in Nondestructive Testing by Eddy Current
}

\author{
Anh-Tuan Phung, Patrice Labie, Olivier Chadebec, Yann Le Floch, \\ and Gérard Meunier
}

\begin{abstract}
In this paper, an application of the automatic cut algorithm applied to nondestructive testing (NDT) is proposed. The $T_{0}-T-\Phi$ finite elements formulation based on the scalar magnetic potential is used in our application. This formulation requires the creation of cut in order to compute the eddy current density in multiply connected conducting region. However, the generation of these cuts can be difficult for the user, particularly in the case of complex geometries. In order to improve robustness of NDT modeling, we proposed an algorithm generating these cuts automatically.
\end{abstract}

\section{Introduction}

Eddy current inspection is a NDT technique using the induced alternating current generated by a emitting coil. Changes in the flow of eddy currents caused by cracks, dimensional variations, or changes in the material's conductivity can be detected by a receiving coil. Let us notice that is some applications, these both coils can be the same. EC-NDT is particularly sensitive to detect small and near surface defects.

This technique has been widely used in inspection and maintenance in the power generation and aircraft industries. Because of the high cost and fault consequences, electromagnetic simulations are intensively used to improve the robustness of NDT device.

In finite elements method, two main formulations can be used to solve eddy currents problems: $\mathrm{A}-\mathrm{V}$ formulation and $\mathrm{T}-\mathrm{T}_{0}-\Phi$ formulation $[1,2]$. This paper

\footnotetext{
A.-T. Phung, P. Labie, O. Chadebec, and G. Meunier Grenoble Electrical Engineering Lab (G2Elab), UMR 5269 INPG-UJF-CNRS, ENSIEG, BP 46, 38402 Saint-Martin-d'Hères Cedex, France Gerard.Meunier@G2elab.Inpg.Fr

Y.L. Floch

Cedrat 15, Chemin de Malacher, Innovallée, 38246 Meylan, France
} 
deals with $\mathrm{T}-\mathrm{T}_{0}-\Phi$ formulation which is known as powerful one in term of computation cost and memory requirement. However, its utilization needs some slight precautions. More precisely, in order to compute current distribution in conducting media around a crossing crack, an artificial cut needs to be created. The automatic cut generation algorithm introduced in this paper avoids users to define these cuts manually. Hence it makes the $\mathrm{T}-\mathrm{T}_{0}-\Phi$ formulation easier to use and the evaluation more reliable.

\section{$2 T_{0}-T-\Phi$ Formulation for EC-NDT}

We consider a typical example in Fig. 1 . In magnetic part $\left(\Omega_{1}\right)$ without current source $(\mathrm{J} \mathrm{s}=0)$, the Maxwell-Ampère equation is written as:

$$
\operatorname{rot} \mathbf{H}=0 \text {. }
$$

It means that the magnetic field can be derived from gradient of a scalar potential $\Phi$ :

$$
\mathbf{H}=-\operatorname{grad}(\Phi) .
$$

To describe the current source $\mathrm{J}_{\mathrm{S}}$, a special source potential $\mathbf{T}_{0}$ is introduced such that:

$$
\operatorname{rot} \mathbf{T}_{0}=J_{S} .
$$

Hence, the magnetic field in air $\left(\Omega_{0}\right)$ is called reduced to this $\mathbf{T}_{0}$ :

$$
\mathbf{H}=\mathbf{T}_{0}-\operatorname{grad}(\Phi)
$$

The $\mathbf{T}_{0}$ calculation must be done in region $\Omega_{\mathrm{To}}$ that encloses excitation coils, to respect Ampere's law. In order to represent induced current in the conductive part $\left(\Omega_{\mathrm{c}}\right)$, from the current conservation law $\operatorname{div} \mathbf{J}=0$, we have $\mathbf{J}=\operatorname{rot} \mathbf{T}$. The field expression is then written as $\mathbf{H}=\mathbf{T}-\operatorname{grad}(\Phi)$. Edge elements are used because their good physical representation of the phenomena. As a result, Maxwell-Ampere

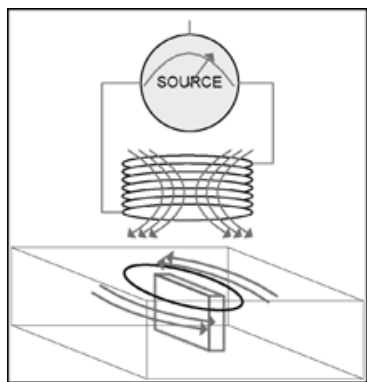

a

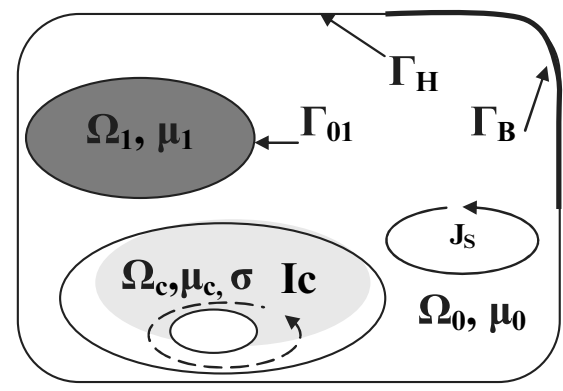

b

Fig. 1 a Typical EC-NDT problem. b Typical schematic problem 
equation is naturally respected. We have to solve the weak form of Maxwell-Gauss and Maxwell-Faraday equations:

$$
\begin{aligned}
& \operatorname{div}\left(-\mu_{1} \operatorname{grad}(\Phi)\right)=0 \text { in } \Omega_{1}, \\
& \operatorname{div}\left(\mu_{0} \mathbf{T}_{0}-\mu_{0} \operatorname{grad}(\Phi)\right)=0 \text { in } \Omega_{0}, \\
& \operatorname{div}\left(\mu_{C} \mathbf{T}-\mu_{C} \operatorname{grad}(\Phi)\right)=0 \text { in } \Omega_{C}, \\
& \text { and } \operatorname{rot}\left(\frac{1}{\sigma} \operatorname{rot} \mathbf{T}\right)+\frac{\partial}{\partial t}\left(\mu_{C}(\mathbf{T}-\operatorname{grad}(\Phi))\right)=0 \text { in } \Omega_{C} .
\end{aligned}
$$

If the conductive parts contain crossing holes, eddy current $I_{C}$ will develop around them. The conductive parts will become multiply connected or closed solid conductors. Hence, the above formulation must be modified in order to take into account this latter induced current $I_{C}$. If we call $\mathbf{j}_{0 \mathrm{C}}$ an arbitrary current density of a prescribed net current circulating around holes and $\mathbf{t}_{0 \mathrm{C}}$ a special vector potential which verifies $\mathbf{j}_{0 C}=\operatorname{rot} \mathbf{t}_{0 C}$ and $\mathbf{t}_{0 C} \times \mathrm{n}=0$ on a domain $\Omega_{0 C}$ surrounding $\Omega_{C}$. Thus, we can choose $\Omega_{0 \mathrm{C}}$ as the union between $\Omega_{0}$ and $\Omega_{\mathrm{C}}$. The magnetic field expression in $\left(\Omega_{\mathrm{c}}\right)$ is written as:

$$
\mathbf{H}=I_{C} \mathbf{t}_{0 C}+\mathbf{T}-\operatorname{grad}(\Phi) .
$$

The current density expression is:

$$
\mathbf{J}=\operatorname{rot}\left(\mathbf{T}+I_{C} \mathbf{t}_{0 C}\right) .
$$

In air region $\left(\Omega_{0}\right)$, the field expression is reduced to this induced current:

$$
\mathbf{H}=I_{C} \mathbf{t}_{0 C}+\mathbf{T}_{0}-\operatorname{grad}(\Phi) .
$$

Let us have a look to the Ampere's theorem. Along any closed path $\mathrm{C}_{1}$ which is entirely in the air and going through the hole, we suppose there is no source current going through surface enclosed by $\mathrm{C}_{1}$. We have in this case:

$$
\int_{C_{1}} \mathbf{H} d l=\int_{C_{1}}\left(I_{C} \mathbf{t}_{0 C}+\mathbf{T}_{0}-\operatorname{grad}(\Phi)\right) d l=I_{C} .
$$

The second term in the right-hand side is equal to zero due to the above condition; the third one vanishes thanks to nature of scalar potential. Finally, Ampere's theorem is verified.

If there are $k$ holes, the presented formulation can be generalized to take into account the $k$ currents circulating around holes. In that case:

$$
\begin{aligned}
\mathbf{H} & =\sum_{k} I_{C k} \mathbf{t}_{0 C k}+\mathbf{T}-\operatorname{grad}(\Phi) \text { in conducting region }\left(\Omega_{c}\right), \\
\mathbf{J} & =\operatorname{rot}\left(\mathbf{T}+\sum_{k} I_{C k} \mathbf{t}_{C 0 k}\right), \\
\mathbf{H} & =\sum_{k} I_{C k} \mathbf{t}_{0 C k}+\mathbf{T}_{0}-\operatorname{grad}(\Phi) \text { in air region }\left(\Omega_{0}\right) .
\end{aligned}
$$


Analyzing the field expression in this formulation, we have to clarify some points. Firstly, the induced current $I_{\mathrm{Ck}}$ is an unknown and must be linked to the total current in the solid conductor by an additional relation $[3,4]$. Secondly, the computation of $\mathbf{t}_{0 \mathbf{c k}}$ could be carried out thanks pre-processing computations. Firstly, an electrokinetic's computation is provided. This could be achieved by imposing electric potential differences on a virtual cut in the multiply connected conductor and then compute the current density $\mathbf{j}_{0 \mathrm{k}}$. Secondly, computation of $\mathrm{t}_{0 \mathrm{CK}}$ can be achieved using the relation between $\mathbf{j}_{0 \mathrm{k}}$ and $\mathbf{t}_{0 \mathrm{k}}$ mentioned above. Once this information is known, the general solution can be carried out without further consideration of the cut. Interested readers are referred to more detailed literature in $[4,5]$. We will here concentrate on the automatic generation and specific treatment of this cut.

This cut is an artificial handle in order to compute the current density joc. In electromagnetic simulation, asking users to manually cut their multi-connected conductor during the modeling process is not consistent. Moreover, if users do not know how to create the right cuts, the obtained results could be completely incorrect. This could arise from complicated conductor's configurations with more than one hole for instance. Hence it is evident that we must automate the creation of these cuts and the linked current density $\mathbf{j}_{0 \mathrm{C}}$ computation.

\section{Automatic Cut Algorithm - Application in NDT}

The basic idea of creating the cut automatically is modeling the inflation of a virtual balloon in a hollow torus. The balloon is kept simply connected by increasing continuously its volume. This process is bounded because the torus volume is limited. This means at the final phase of the process, joining part of the balloon's boundary must form the required cuts [6].

Cuts are modeled by shell elements with double nodes having the same coordinates. Electric scalar potential are imposed on both sides of cuts to create the current distribution. Values of electric potential can be chosen arbitrarily and scaled to lead to a global unit current.

The algorithm is implemented and tested in a standard the finite elements code Flux [7] and lead to excellent results in some cases. In other applications, for example in NDT problems, this algorithm reveals a major drawback. In fact, NDT geometries contain one or more very thin cracks - crossing or not. When applying inflating balloon algorithm to these geometries, it can lead to "T-shaped" cuts (see Fig. 2).

This situation occurs because we did not implement any control on the inflating direction in the algorithm. Hence, balloon's boundaries developed themselves freely in all directions and they could meet each other. From a topological point of view, these crossing cuts cause any trouble because they effectively divide the multiply connected volume into simply connected one. All holes are linked to outward by these cuts.

But from a modeling point of view, these cuts are not straightforward to work with. In fact, on the junction of more than one branch of cuts, values of electric 


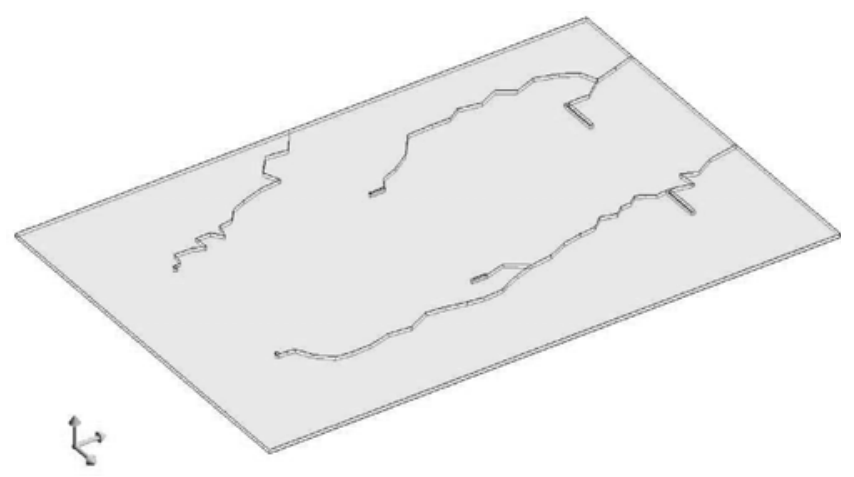

Fig. 2 Crossing automatic cuts
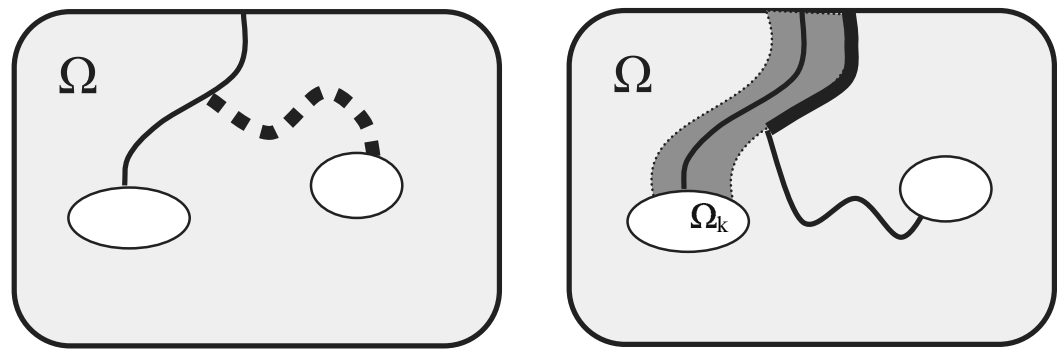

Fig. 3 "T-shaped" cut and protection wall around existing cut redirects future cut to exterior

potential can not be simply affected in existing formulations. Currently, these kinds of "T shaped" cuts leads to a failure $\mathbf{j}_{0 \mathrm{C}}$ computation.

This problem can be solved by modifying the numerical implementation of shell elements allowing the management of scalar potential value on nodes located in "T-shaped" area. Potential jumps must be attributed depending on number of branches at each junction. However, this solution is difficult to implement and we prefer to control the number of cuts created after inflating process and force the algorithm to create a separated cut for each hole.

This solution has been implemented with the following strategy. First of all, "T-shaped" cuts are considered and an extra branch is eliminated for each T to get simple cuts. The procedure is repeated until no more "T-shaped" will be found. At the end of this step, cuts are missing in comparison with the number of holes. In the following, 2D model and previous notations are used to facilitate the comprehension.

Inflating algorithm is the applied one more time but existing cuts have to be protected to prevent new cuts to join the old ones. If we call $\Omega$ the whole domain and $\mathrm{C}_{\mathrm{K}}$ any existing cut, we form a special bounded sub-domain $\Omega_{\mathrm{K}}$ for each $\mathrm{C}_{\mathrm{K}}$. If a future cut plugs into these $\Omega_{K}$, they will be redirected to exterior or holes (see Fig. 3) hence preventing formation of crossing cuts. This is repeated until the number of cuts is equal to the number of holes. 
Fig. 4 Formation mechanism of separated sub-domain
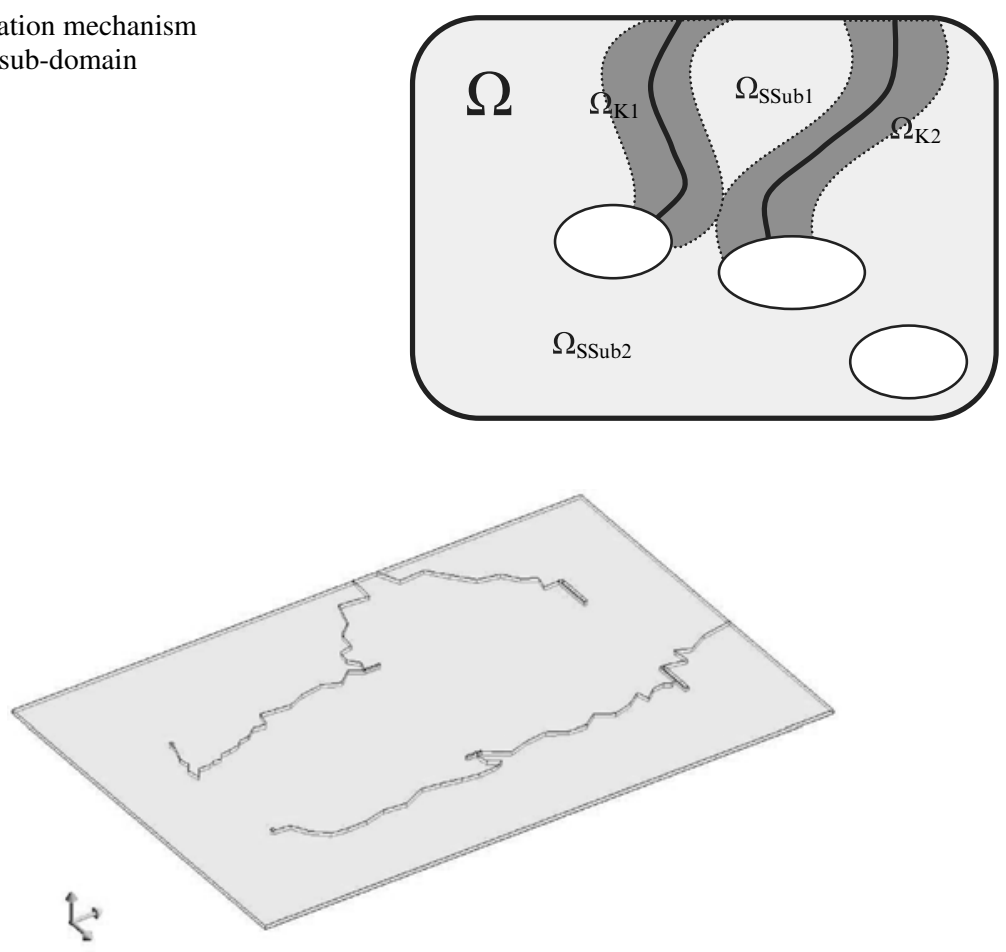

Fig. 5 Automatic cuts after treatment

One can observe that $\Sigma \Omega_{\mathrm{K}} \neq \Omega$, or $\Omega / \Sigma \Omega_{\mathrm{K}} \neq \varnothing$. This means in some cases, linked protected zones can divide the great domain into separated sub-domains $\Omega_{\text {SSub }}$. Hence inflating algorithm will have some difficulties to generate correct number of cuts. This situation is occurring when dealing with low-meshed geometries in which sub-domains $\Omega_{\mathrm{K}}$ have more chances to touch each other (see Fig. 4 ). To get rid of this problem, initialization phase of inflating algorithm must be carefully prepared. It must permit initial element having random walk onto $\Omega_{\mathrm{SSub}}$ so that all positions could be scanned by the algorithm. This technique has been implemented in our work and gives interesting results (see Fig. 5).

We present here some computation results on a NDT test case with six crossingcracks on a $1 \mathrm{~mm}$ thick plate (conductivity $59.6 \times 10^{6}$ siemens). The probe is an air-core type one and is located at $5 \mathrm{~mm}$ above the plate. It is fed with a current source at $100 \mathrm{kHz}$. To demonstrate the robustness of the automatic cut algorithm, we will compute the global inductance value in both cases of manual cut and the automatic one.

Figure 6 shows vector field of induced current in the conductive plate. Crossing cuts detected on Fig. 2 are replaced automatically with separated ones. All holes are linked to outside. Eddy current circulation is properly computed. 


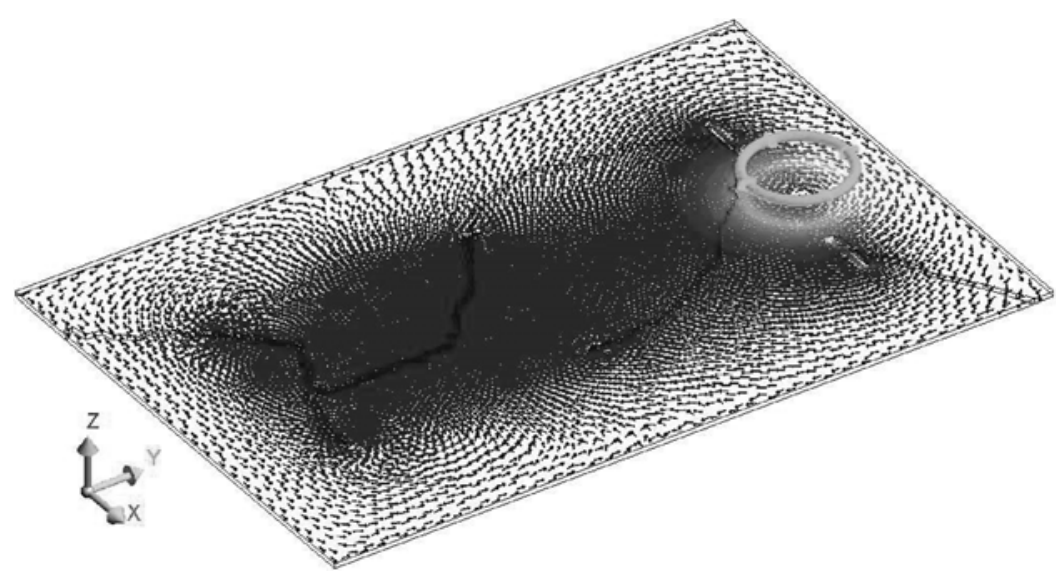

Fig. 6 Eddy current vector field on the conductive plate with automatic cuts

Table 1 Computation comparison

\begin{tabular}{lccc}
\hline Result in case of & Manual cuts & Automatic cuts & Relative error \\
\hline Losses in the plate & $57.42 \mu \mathrm{W}$ & $55.62 \mu \mathrm{W}$ & $3.13 \%$ \\
Coil inductance & $46.69 \mu \mathrm{H}$ & $45.58 \mu \mathrm{H}$ & $2.37 \%$ \\
\hline
\end{tabular}

In Table 1, we presents losses and coil inductance in both cases. A slight difference between two results can be observed. This must be principally due to the mesh variation between two cases.

\section{Conclusion}

The application of automatic cuts algorithm with some adapted treatments for ECNDT geometries leads to an easier use of $\mathrm{T}_{0}-\mathrm{T}-\Phi$ formulation. Computation result is comparable between manual and automatic cut. The creation of cuts is user-free and contributes to the enhancement of $\mathrm{T}-\mathrm{T}_{0}-\Phi$.

\section{References}

1. Biro O., Preis K., Renhart W., Vrisk G., Richter K.R., Computation of 3-D current driven skin effect problems using a current vector potential, IEEE Transactions on Magnetics, 29(2), pp. 1325-1328, Mar. 1993.

2. Bouissou S., Piriou F., Kieny C., and Tanneau G., A numerical simulation of a power transformer using 3-D finite element method coupled to circuit equation, IEEE Transactions on Magnetics, 30(5), pp. 3224-3227, Sep. 1994. 
3. Bedrosian G., Magnetostatic cancellation error revisited, IEEE Transactions on Magnetics, 27(5), pp. 4181-4184, Sep. 1991.

4. Meunier G., Luong H.T., and Marechal Y., Computation of coupled problem of 3D eddy current and electrical circuit by using T0 $-\mathrm{T}-\varphi$ formulation, IEEE Transactions on Magnetics, 34(5), Part 1, pp. 3074-3077, Sept. 1998.

5. LeFloch Y., Meunier G., Guerin C., Labie P., Brunotte X., and Boudaud D., Coupled problem computation of 3-D multiply connected magnetic circuits and electrical circuits, IEEE Transactions on Magnetics, 39(3), Part 1, pp. 1725-1728, May 2003.

6. Phung A.T., Chadebec O., Labie P., Le Floch Y., and Meunier G., Automatic cuts for magnetic scalar potential formulations, IEEE Magnetics Transactions, 41(5), pp. 1668-1671, May 2005.

7. Flux Software (http://www.cedrat.com) 